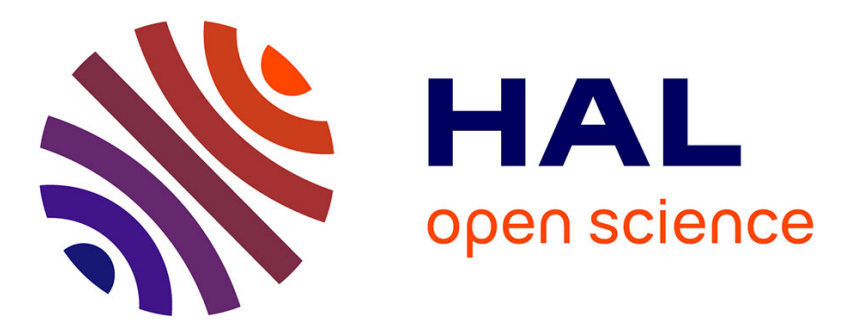

\title{
Mechanism of the quasi-zero axial acoustic radiation force experienced by elastic and viscoelastic spheres in the field of a quasi-Gaussian beam and particle tweezing
}

F. G. Mitri, Zine El Abiddine Fellah

\section{- To cite this version:}

F. G. Mitri, Zine El Abiddine Fellah. Mechanism of the quasi-zero axial acoustic radiation force experienced by elastic and viscoelastic spheres in the field of a quasi-Gaussian beam and particle tweezing. Ultrasonics, 2013, 54 (1), pp.351-357. 10.1016/j.ultras.2013.04.010 . hal-00813136

\author{
HAL Id: hal-00813136 \\ https://hal.science/hal-00813136
}

Submitted on 15 Apr 2013

HAL is a multi-disciplinary open access archive for the deposit and dissemination of scientific research documents, whether they are published or not. The documents may come from teaching and research institutions in France or abroad, or from public or private research centers.
L'archive ouverte pluridisciplinaire HAL, est destinée au dépôt et à la diffusion de documents scientifiques de niveau recherche, publiés ou non, émanant des établissements d'enseignement et de recherche français ou étrangers, des laboratoires publics ou privés. 
1 Mechanism of the quasi-zero axial acoustic radiation

2 force experienced by elastic and viscoelastic spheres in

3 the field of a quasi-Gaussian beam and particle tweezing

4

5 F.G. Mitri ${ }^{\dagger}$, Z.E.A. Fellah*

$6{ }^{\dagger}$ Los Alamos National Laboratory, MPA-11, Sensors \& Electrochemical Devices,

7 Acoustics \& Sensors Technology Team, MS D429, Los Alamos, NM 87545, USA

8

9 *Laboratoire de Mécanique et d'Acoustique, CNRS-UPR 7051, 31 chemin Joseph

10 Aiguier, Marseille, 13402, France.

11

13 Corresponding author:

14 F.G. Mitri, emails: mitri@,ieee.org, mitri@lanl.gov 
1 Abstract - The present analysis investigates the (axial) acoustic radiation force induced

2 by a quasi-Gaussian beam of progressive (traveling) waves centered on an elastic and a

3 viscoelastic (polymer-type) sphere in a nonviscous fluid. The quasi-Gaussian beam is an

4 exact solution of the source free Helmholtz wave equation and is characterized by an

5 arbitrary waist $w_{0}$ and a diffraction convergence length known as the Rayleigh range $z_{R}$.

6 Examples are found where the radiation force unexpectedly approaches closely to zero at

7 some of the elastic sphere's resonance frequencies for $k w_{0} \leq 1$ (where this range is of

8 particular interest in describing strongly focused or divergent beams), which may produce

9 particle immobilization along the axial direction. Moreover, the (quasi)vanishing

10 behavior of the radiation force is found to be correlated with conditions giving extinction

11 of the backscattering by the quasi-Gaussian beam. Furthermore, the mechanism for the

12 quasi-zero force is studied theoretically by analyzing the contributions of the kinetic,

13 potential and momentum flux energy densities and their density functions. It is found that

14 all the components vanish simultaneously at the selected $k a$ values for the nulls.

15 However, for a viscoelastic sphere, acoustic absorption degrades the quasi-zero mean

16 force. 


\section{1. Introduction}

2 Quasi-Gaussian beams have been recently originated in the wave diffraction theory as an

3 exact solution of the Helmholtz equation. The properties of such beams have been

4 analyzed from the standpoint of the classical wave propagation theory based on the

5 complex source point method [1-8] to obtain the expression of the pressure for the

6 incident quasi-Gaussian beam, and expand it using a partial-wave series $[9,10]$. A quasi-

7 Gaussian beam (Fig. 1) is characterized by an arbitrary waist $w_{0}$ and a diffraction

8 convergence length known as the Rayleigh range $z_{R}$. Moreover, the beam has the form

9 of a superposition of sources and sinks with complex coordinates [9].

10 In a recent investigation [11], the scattering (which is an important phenomenon in 11 many applications, for example nondestructive imaging applications [12, 13], medical

12 imaging etc.), instantaneous and mean radiation forces experienced by a rigid and

13 immovable (fixed) sphere centered on the axis of the beam have been investigated

14 theoretically. Situations have been observed where significant differences have occurred

15 between the quasi-Gaussian beam and the plane wave results for $k w_{0}<25$, (where $k$

16 denotes the wavenumber of the incident beam), however, the plane wave results have

17 been recovered when $k w_{0}>25$ and increases toward $\rightarrow \infty$.

18 The purpose here is to illustrate situations where the radiation force function (which

19 the radiation force per unit energy density and unit cross-section) tends to zero at some of

20 the resonance frequencies of an elastic sphere and specific values of $k w_{0}$. The formalism

21 for the scattering derived previously [11] is used here to evaluate the acoustic radiation

22 force of a quasi-Gaussian beam on an elastic sphere in a nonviscous fluid, and correlate

23 the backscattering and radiation force function plots. Moreover, the mechanism for the 
1 quasi-zero force is studied theoretically by analyzing the contributions of the kinetic,

2 potential and momentum flux energy densities and their density functions. Additional

3 examples are provided for a (polymer-type) viscoelastic sphere. The extension of the

4 previous work [11] to account for the sphere's elasticity may be helpful for the

5 identification of some conditions where ultrasonic quasi-Gaussian beams may be used to

6 immobilize a sphere (or a spherical shell, a layered sphere [14-16], or a layered spherical

7 shell [17]) in a fluid with negligible viscosity. It is important to identify such conditions

8 using a priori information obtained from theoretical predictions since it may be

9 experimentally easier to verify the existence of zero acoustic radiation forces in quasi-

10 Gaussian beams using solid objects.

11

\section{2. Radiation force, its components and density functions}

13 The mean (time-averaged) radiation force of a quasi-Gaussian beam of continuous waves

14 is expressed as [18, 19],

$$
\left\langle\mathbf{F}_{r a d}\right\rangle=\iint_{S_{0}}\langle\mathscr{L}\rangle \mathbf{n} d S-\iint_{S_{0}}\left\langle\rho \mathbf{v}^{(1)}\left(\mathbf{v}^{(1)} \cdot \mathbf{n}\right)\right\rangle d S,
$$

16 where,

$$
\begin{aligned}
\mathscr{L} & =\frac{\rho_{0}}{2}\left|\mathbf{v}^{(1)}\right|^{2}-\frac{1}{2 \rho_{0} c_{0}^{2}} p^{(1)^{2}} \\
& =\mathscr{K}-\mathscr{U},
\end{aligned}
$$

18 is the Lagrangean energy density, the superscript ${ }^{(1)}$ denotes first-order quantities,

$19 \mathbf{v}^{(1)}=\nabla \varphi, \quad p^{(1)}=-\rho_{0} \frac{\partial \varphi^{(1)}}{\partial t}$, and $\varphi^{(1)}=\operatorname{Re}\left[\Phi^{(1)}\right]$, where $\Phi^{(1)}$ is the total (incident + 
1 scattered) linear velocity potential that is related to the total pressure in the surrounding

2 fluid.

3 This equation can be rewritten in terms of the following factors [20],

$$
\left\langle\mathbf{F}_{r a d}\right\rangle=\iint_{S_{0}}\langle\mathscr{\mathscr { C }}\rangle \mathbf{n} d S-\iint_{S_{0}}\langle\mathscr{Q}\rangle \mathbf{n} d S-\iint_{S_{0}}\langle\mathscr{P}\rangle d S
$$

5 where $\mathscr{\mathscr { R }}=\rho_{0} \mathbf{v}^{(1)} v_{n}^{(1)}$, is the momentum flux energy density, and $v_{n}^{(1)}$ is the normal

6 component of the velocity. The three components of the radiation force on an elastic

7 sphere can be represented in terms of the total velocity potential $\Phi^{(1)}$ given by the

8 partial-wave series as,

$$
\varphi^{(1)}=\operatorname{Re}\left[\Phi^{(1)}\right]=\sum_{n=0}^{\infty} \Phi_{0}(2 n+1) R_{n} P_{n}(\cos \theta),
$$

10 where, $\Phi_{0}$ is the amplitude. The coefficient $R_{n}$ is given by [11],

$$
R_{n}=\operatorname{Re}\left[i^{n}\left(U_{n}(k r)+i V_{n}(k r)\right) g_{n}\left(k z_{R}\right) e^{-i \omega t}\right],
$$

12 and,

$$
\begin{aligned}
U_{n} & =\left(1+\alpha_{n}\right) j_{n}(k r)-\beta_{n} y_{n}(k r), \\
V_{n} & =\beta_{n} j_{n}(k r)+\alpha_{n} y_{n}(k r),
\end{aligned}
$$

14 where $y_{n}($.$) are the spherical Neumann functions (or the spherical Bessel functions of$

15 the second kind), $\alpha_{n}=\operatorname{Re}\left[S_{n}\right], \quad \beta_{n}=\operatorname{Im}\left[S_{n}\right]$, and $S_{n}$ are the scattering coefficients

16 determined by applying appropriate boundary conditions at the interface fluid-structure,

17 with the assumption that the surrounding fluid is nonviscous. These functions depend on

18 the sphere's elastic parameters such as the longitudinal $c_{L}$, the shear or transverse $c_{T}$

19 sound speed and the mass densities of both the fluid $\rho_{0}$ and the sphere $\rho_{s}$. It should be 
1 emphasized that those coefficients are found equivalent to those obtained from the study

2 of acoustic scattering by plane waves (See Appendix in [21]).

3 The three components of the radiation force are now expressed as [20],

4

$$
\begin{aligned}
\iint_{S_{0}}\langle\mathscr{K}\rangle \mathbf{n} d S & =\pi \rho_{0} a^{2}\left(\frac{1}{a^{2}} \int_{0}^{\pi}\left\langle\left(\frac{\partial \Phi^{(1)}}{\partial \theta}\right)_{r=a}^{2}\right\rangle \sin \theta \cos \theta d \theta+\int_{0}^{\pi}\left\langle\left(\frac{\partial \Phi^{(1)}}{\partial r}\right)_{r=a}^{2}\right\rangle \sin \theta \cos \theta d \theta\right) \\
& =2 \pi \rho_{0}\left|\Phi_{0}\right|^{2} \sum_{n=0}^{\infty}\left\{g_{n}\left(k z_{R}\right) g_{n+1}\left(k z_{R}\right)(n+1)\left[\begin{array}{c}
n(n+2)\left(V_{n} U_{n+1}-U_{n} V_{n+1}\right) \\
+(k a)^{2}\left(V_{n}^{\prime} U_{n+1}^{\prime}-U_{n}^{\prime} V_{n+1}^{\prime}\right)
\end{array}\right]\right\}_{r=a},
\end{aligned}
$$

5

$$
\begin{gathered}
\iint_{S_{0}}\langle\mathscr{Q}\rangle \mathbf{n} d S=\frac{\pi \rho_{0} a^{2}}{c_{0}^{2}} \int_{0}^{\pi}\left\langle\left(\frac{\partial \Phi^{(1)}}{\partial t}\right)_{r=a}^{2}\right\rangle \sin \theta \cos \theta d \theta \\
=2 \pi \rho_{0}\left|\Phi_{0}\right|^{2}(k a)^{2} \sum_{n=0}^{\infty}\left\{g_{n}\left(k z_{R}\right) g_{n+1}\left(k z_{R}\right)(n+1)\left(V_{n} U_{n+1}-U_{n} V_{n+1}\right)\right\} \\
\iint_{S_{0}}\langle\mathscr{R}\rangle d S=-2 \pi \rho_{0} a^{2}\left(\frac{1}{a} \int_{0}^{\pi}\left\langle\left(\frac{\partial \Phi^{(1)}}{\partial r}\right)_{r=a}\left(\frac{\partial \Phi^{(1)}}{\partial \theta}\right)_{r=a}\right) \sin ^{2} \theta d \theta+\int_{0}^{\pi}\left\langle\left(\frac{\partial \Phi^{(1)}}{\partial r}\right)_{r=a}^{2}\right) \sin \theta \cos \theta d \theta\right) \\
=-2 \pi \rho_{0} k a\left|\Phi_{0}\right|^{2} \sum_{n=0}^{\infty}\left\{\begin{array}{l}
g_{n}\left(k z_{R}\right) g_{n+1}\left(k z_{R}\right)(n+1) \\
\left.\left[\begin{array}{l}
n\left(V_{n} U_{n+1}^{\prime}-U_{n} V_{n+1}^{\prime}\right)-(n+2)\left(V_{n}^{\prime} U_{n+1}-U_{n}^{\prime} V_{n+1}\right) \\
-2(k a)\left(V_{n}^{\prime} U_{n+1}^{\prime}-U_{n}^{\prime} V_{n+1}^{\prime}\right)
\end{array}\right]\right\}_{r=a}
\end{array}\right.
\end{gathered}
$$

6

7

8 Denoting by $E=\rho k^{2}\left|\Phi_{0}\right|^{2} / 2$ the characteristic energy density, the axial time-

9 averaged radiation force of a quasi-Gaussian beam is expressed by [11],

10

$$
\left\langle F_{z, r a d}\right\rangle=Y_{q G} S_{c} E
$$

11 where $S_{c}=\pi a^{2}$ is the cross-sectional area, and $Y_{q G}$ is the radiation force function, which

12 is the radiation force per unit energy density and unit cross-sectional surface given by 13 [11], 


$$
Y_{q G}=-\frac{4}{(k a)^{2}} \sum_{n=0}^{\infty}\left\{g_{n}\left(k z_{R}\right) g_{n+1}\left(k z_{R}\right)(n+1)\left[\alpha_{n}+\alpha_{n+1}+2\left(\alpha_{n} \alpha_{n+1}+\beta_{n} \beta_{n+1}\right)\right]\right\} .
$$

2 In the same manner, the form functions for the kinetic energy density $K_{q G}$, potential

3 energy density $U_{q G}$ and momentum flux density $R_{q G}$ are defined as,

4

$$
\begin{aligned}
K_{q G}= & \frac{\iint_{S_{0}}\langle\mathscr{\mathscr { C }}\rangle \mathbf{n} d S}{\pi a^{2} E} \\
= & \frac{4}{(k a)^{2}} \sum_{n=0}^{\infty}\left[g_{n}\left(k z_{R}\right) g_{n+1}\left(k z_{R}\right)(n+1)\left\{n(n+2)\left(V_{n} U_{n+1}-U_{n} V_{n+1}\right)+(k a)^{2}\left(V_{n}^{\prime} U_{n+1}^{\prime}-U_{n}^{\prime} V_{n+1}^{\prime}\right)\right\}\right]_{r=a},
\end{aligned}
$$

5

6

$$
\begin{aligned}
& \iint_{S_{0}}\langle\mathscr{Q}\rangle \mathbf{n} d S \\
U_{q G}= & \pi a^{2} E \\
= & -4 \sum_{n=0}^{\infty}\left\{g_{n}\left(k z_{R}\right) g_{n+1}\left(k z_{R}\right)(n+1)\left(V_{n} U_{n+1}-U_{n} V_{n+1}\right)\right\}_{r=a},
\end{aligned}
$$

7

$$
R_{q G}=-\frac{\iint_{S_{0}}\langle\mathscr{\mathscr { P }}\rangle d S}{\pi a^{2} E}
$$

$$
=\frac{4}{(k a)} \sum_{n=0}^{\infty}\left\{g_{n}\left(k z_{R}\right) g_{n+1}\left(k z_{R}\right)(n+1)\left[\begin{array}{l}
n\left(V_{n} U_{n+1}^{\prime}-U_{n} V_{n+1}^{\prime}\right)-(n+2)\left(V_{n}^{\prime} U_{n+1}-U_{n}^{\prime} V_{n+1}\right) \\
-2(k a)\left(V_{n}^{\prime} U_{n+1}^{\prime}-U_{n}^{\prime} V_{n+1}^{\prime}\right)
\end{array}\right]\right\}_{r=a},
$$

8

9 so the radiation force function is rewritten as,

$$
Y_{q G}=K_{q G}+U_{q G}+R_{q G}
$$

11 To further calculate the radiation force function's distribution versus the polar angle $\theta$

12 over the sphere's surface at a particular dimensionless frequency $k a$, a density function

$13 y_{q G}(\theta)$ is defined for $Y_{q G}$ as,

$$
Y_{q G}=\int_{0}^{\pi} y_{q G}(\theta) d \theta
$$


1 The density function $y_{q G}(\theta)$ physically represents the contribution of the radiation force

2 function along a certain direction $\theta$. Following Eq.(16), the kinetic $k_{q G}(\theta)$, potential

$3 u_{q G}(\theta)$ and momentum flux $r_{q G}(\theta)$ density functions are defined as,

4

5

6

7 The density form functions are expressed as,

$$
K_{q G}=\int_{0}^{\pi} k_{q G}(\theta) d \theta,
$$

$$
U_{q G}=\int_{0}^{\pi} u_{q G}(\theta) d \theta,
$$

$$
R_{q G}=\int_{0}^{\pi} r_{q G}(\theta) d \theta
$$

$$
k_{q G}(\theta)=\frac{\rho_{0}}{E}\left(\frac{1}{a^{2}}\left\langle\left(\frac{\partial \Phi^{(1)}}{\partial \theta}\right)_{r=a}^{2}\right\rangle+\left\langle\left(\frac{\partial \Phi^{(1)}}{\partial r}\right)_{r=a}^{2}\right\rangle\right) \sin \theta \cos \theta,
$$

$$
u_{q G}(\theta)=-\frac{\rho_{0}}{c_{0}^{2} E}\left\langle\left(\frac{\partial \Phi^{(1)}}{\partial t}\right)_{r=a}^{2}\right\rangle \sin \theta \cos \theta,
$$

10

$$
r_{q G}(\theta)=2 \frac{\rho_{0}}{E}\left(\frac{1}{a}\left\langle\left(\frac{\partial \Phi^{(1)}}{\partial r}\right)_{r=a}\left(\frac{\partial \Phi^{(1)}}{\partial \theta}\right)_{r=a}\right\rangle \sin ^{2} \theta-\left\langle\left(\frac{\partial \Phi^{(1)}}{\partial r}\right)_{r=a}^{2}\right\rangle \sin \theta \cos \theta\right) .
$$

11 Using the identity for the time-average of a product of two complex functions (p. 25-26

12 in [22]), Eqs.(20)-(22) can be directly evaluated at $r=a$ using Eqs.(4)-(6), so that the

13 density function $y_{q G}(\theta)$ for $Y_{q G}$ is expressed as,

$$
y_{q G}(\theta)=k_{q G}(\theta)+u_{q G}(\theta)+r_{q G}(\theta) .
$$




\section{3. Numerical results, discussion and concluding remarks}

2 The following examples are considered to illustrate the theory by plotting the

3 radiation force function $Y_{q G}$ for acoustical quasi-Gaussian beam incident upon elastic and

4 viscoelastic spheres immersed in water $\left(\rho_{\text {water }}=1060 \mathrm{~kg} / \mathrm{m}^{3}, c_{\text {water }}=1470 \mathrm{~m} / \mathrm{s}\right)$. In

5 addition, the magnitude of the backscattering form-function $\left|f_{\infty}\left(k a, k z_{R}, \pi\right)\right|$, (Eq.(8) in

6 [11]) is displayed to correlate the radiation force function plots with the backscattering.

7 The simulations are evaluated in the dimensionless frequency range $0<k a \leq 10$ for

8 selected values of the dimensionless beam waist parameter $k w_{0}$ at which the quasi-zero

9 behavior in the $Y_{q G}$ plots is manifested.

10 The top and bottom panels in Figure 2 show the plots for the backscattering form11 function (Eq.(8) in [11]) and radiation force function (Eq.(11)), respectively, for a

12 polymethylmetacrylate (PMMA) elastic sphere $\left(\rho_{P M M A}=1191 \mathrm{~kg} / \mathrm{m}^{3}, c_{L, P M M A}=2690\right.$

$13 \mathrm{~m} / \mathrm{s}, c_{T, P M M A}=1340 \mathrm{~m} / \mathrm{s}$ ) for $k w_{0}=0.1$ (solid line), $k w_{0}=1$ (long-dashed line), $k w_{0}=1.5$

14 (dashed-dotted line), and $k w_{0}=2$ (dotted line). The arrows along the $k a$ axis in the bottom

15 panel point to the zeros of $Y_{q G}$ that occur at the minima-resonances of the elastic sphere.

16 Visual inspection and comparison of both curves indicate the correlation of the quasi-zero

17 radiation force with the reduction in the backscattering direction; the nulls in the plots for

$18\left|f_{\infty}\left(k a, k z_{R}, \pi\right)\right|$ closely match those of $Y_{q G}$ for $k w_{0} \leq 1$. At those specific $k a$ values that

19 correspond to nulls, the transmission of sound waves through the elastic sphere in the

20 forward direction (i.e. axial direction $\theta=0$ ) is total. Moreover, as explained in [9], this

21 range of $k w_{0} \leq 1$ values is of particular interest in describing strongly focused or 
1 divergent beams. As $k w_{0}$ increases, the magnitude of the backscattering as well as the

2 amplitude of the radiation force function increase.

3 To closely examine the conditions for which the nulls tend to appear (pointed by

4 arrows in Fig. 3) in the plots, the components $K_{q G}, U_{q G}, R_{q G}$ as well as $Y_{q G}$ are evaluated

5 through Eqs.(12)-(15) for $k w_{0}=0.1$. From Fig. 3, it is noticed that all the three

6 components, namely the kinetic energy density, the potential energy density as well as

7 the momentum flux density vanish simultaneously at the selected $k a$ values for the nulls,

8 unlike the case of the zero-force predicted for spherical waves on a rigid sphere (See Fig.

93 in [23], around $k a=3.9$ ) for which both the kinetic energy density as well as the

10 potential energy density have same magnitudes but opposite amplitudes. In addition, it is

11 noticeable that for a tightly focused (or strongly divergent) quasi-Gaussian beam (i.e.,

$\left.12 k w_{0} \leq 1\right)$, though the axial radiation force approaches closely to zero, it is not found to be

13 negative (i.e. not a force of attraction), whereas for some situations, theoretical

14 predictions have demonstrated the existence of a negative (pulling) force on a sphere

15 placed in the close proximity of acoustical spherical waves [23-26], or in the field of

16 focused Gaussian beams [27, 28], Bessel beams [29-32], plane waves on an elastic

17 spherical shell close to a boundary [33], or plane waves on a coated sphere [16].

18 Complete acoustical tweezing requires immobilization of a particle in the acoustical field

19 (i.e. producing a mean zero force). However, in practical cases, a pulling force may be

20 required to counteract the effects of possible mechanical instabilities (e.g., hydrodynamic

21 forces, viscous forces, etc.) that could destabilize the trap using a single beam. Further

22 experiments using acoustical quasi-Gaussian beams are warranted to address this 23 problem. 
1 To analyze the behavior of the radiation force function and its density distribution

2 along a selected direction $\theta$, the kinetic, potential, momentum flux, and radiation force

3 density functions are evaluated using Eqs.(20)-(23) for $k w_{0}=0.1$ at the zeros of

$4 \quad Y_{q G}$ (pointed to by arrows in Fig. 3). The results are displayed in panels (a)-(d) of Fig. 4

5 for $k a=3.366,4.806,6.456$ and 7.691, respectively. In all cases, all the density functions

6 including $y_{q G}(\theta)$ exhibit an anti-symmetric behavior with respect to the direction $\theta / \pi=$

7 0.5. From Fig. 4, one concludes that both anterior $(0 \leq \theta / \pi \leq 0.5)$ and posterior $(0.5 \leq \theta / \pi$

$8 \leq 1)$ areas of the sphere experience a force of equal magnitude in opposite direction,

9 resulting in a zero mean force on the sphere, at the selected $k a$ values.

10 Viscoelasticity inside the sphere's material and its effect on the radiation force

11 function for a quasi-Gaussian beam is further analyzed by introducing complex wave

12 numbers into the theory [34-36]. The curves shown in Fig. 3 for the elastic sphere case,

13 are now computed for a viscoelastic PMMA sphere, for which the plots for the

14 components $K_{q G}, U_{q G}, R_{q G}$ as well as $Y_{q G}$ are shown in Fig. 5 for $k w_{0}=0.1$. For the first

15 null that have occurred at $k a=3.366$ for the elastic sphere case, the inclusion of

16 absorption induces a slight shift to higher $k a$ so that the first minimum in the plot for $Y_{q G}$

17 occurs at $k a=3.406$. Moreover, an increase in the kinetic $K_{q G}$ and potential $U_{q G}$ energy

18 densities counteract the momentum flux density $R_{q G}$, giving birth to a positive (repulsive)

19 force. The third null that have occurred at $k a=6.456$ for the elastic sphere case (Fig. 3),

20 becomes a minimum in the viscoelastic case that is shifted to lower $k a=6.406$. As a

21 general observation, comparison of Figs. 3 and 5 show that absorption degrades the zero-

22 mean force. Initially, this behavior has been observed for the axial radiation force of a

23 zero-order Bessel acoustical beam on a polyethylene viscoelastic sphere (see Fig. 8 in 
1 Ref. [37]), and later discussed to include vortex beams by introducing the notion of

2 acoustical efficiency factors [38].

3 Finally, additional computations are performed to examine the effect of varying $k w_{0}$

4 on the $Y_{q G}$ curves. Fig. 6 shows the plots for a PMMA elastic sphere in water for $k w_{0}=5$,

510 and 25, respectively. As shown previously [11], when $k w_{0} \geq 25$, the $Y_{q G}$ plot closely

6 approaches $Y_{p}$, where $Y_{p}$ is the radiation force function for plane waves [36]. Fig. 6

7 shows that some resonances in the radiation force function curves tend to appear as $k w_{0}$

8 increases. To study this behavior, the magnitude of the backscattering form function

$9\left|f_{\infty}\left(k a, k z_{R}, \pi\right)\right|$ is plotted for the same set of parameters chosen for Fig. 6. Comparison of

10 both figures show that the suppression of the resonance in the radiation force function

11 curve of Fig. 6 around $k a=5.336$ for $k w_{0}=5$, is associated with a reduction in the

12 backscattering direction (Fig. 7). Moreover, the suppression of the $Y_{q G}$-resonances in Fig.

136 around $k a=7.461$ and 8.876 for $k w_{0}=5$, is associated with a suppression of the

14 scattering in the backward direction (Fig. 7). This behavior has also been observed in the

15 context of Bessel beams [29]; that is a reduction of the scattering into the backward

16 hemisphere reduces the radiation force.

17 Concerning the case where the sphere is shifted off-axis of the beam and the issue

18 related to transverse stability, recent investigations based on the partial-wave expansion

19 method [39], and utilizing the arbitrary scattering theory [40-43], have shed some light

20 onto this topic for the case of Bessel beams [44, 45]. Those studies can be potentially

21 extended to the case of quasi-Gaussian beams, and further experimental data is warranted

22 to support the theoretical predictions and demonstrate the feasibility of particle tweezing. 
1 Acknowledgments: The financial support provided through a Director's fellowship

2 (LDRD-X9N9, Project \# 20100595PRD1) from Los Alamos National Laboratory is

3 gratefully acknowledged. Disclosure: this unclassified publication, with the following

4 reference no. LA-UR 13-XXXXX, has been approved for unlimited public release under 5 DUSA ENSCI.

6 
Figures
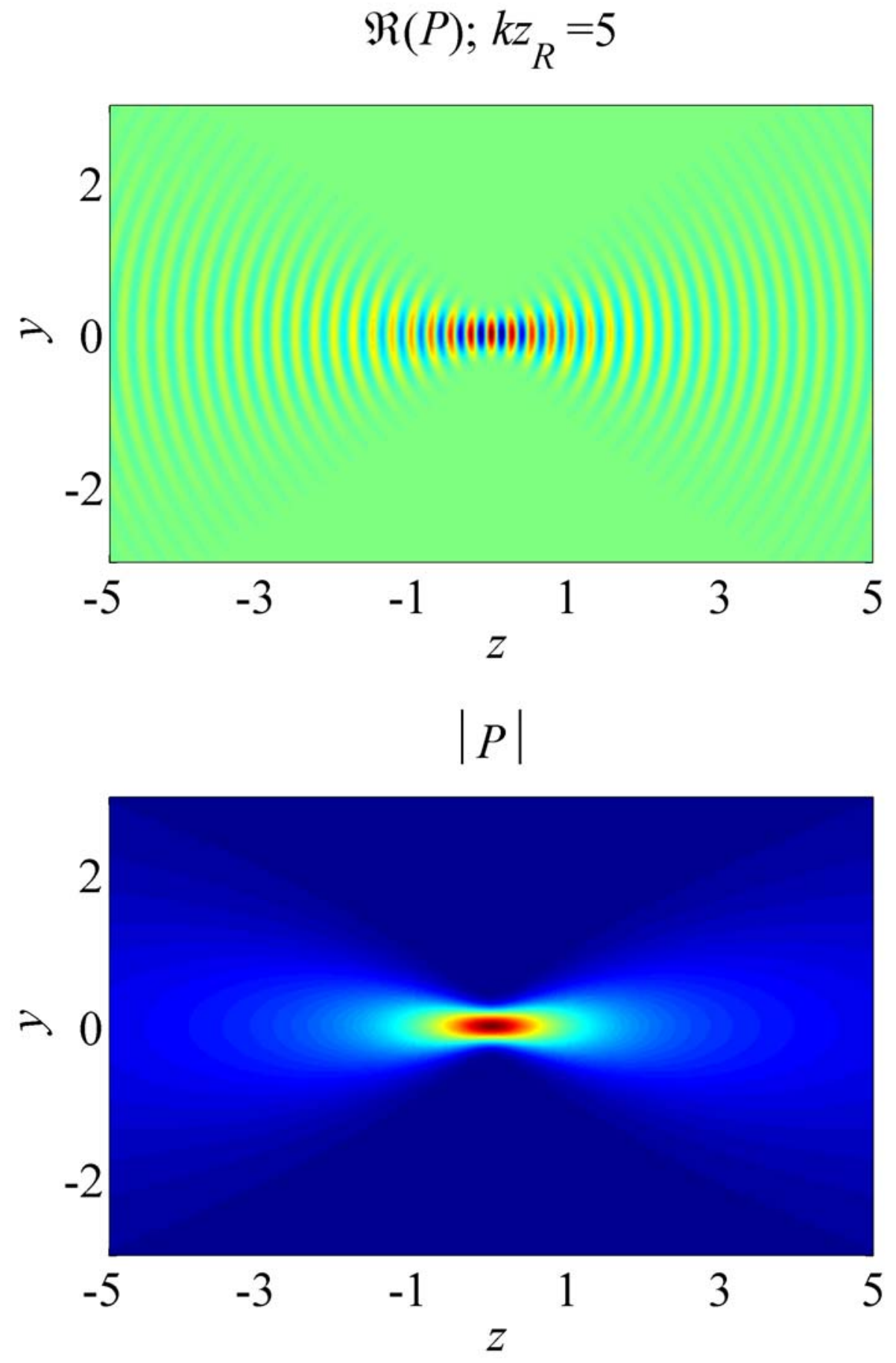

3 Fig. 1. (Color online) Instantaneous sound pressure (top panel) for a quasi-Gaussian beam

4 at $k w_{0}=5$. The bottom panel represents the magnitude of the pressure for $k=25 \mathrm{~m}^{-1}$.

5 (See also the Supplementary Animation). 

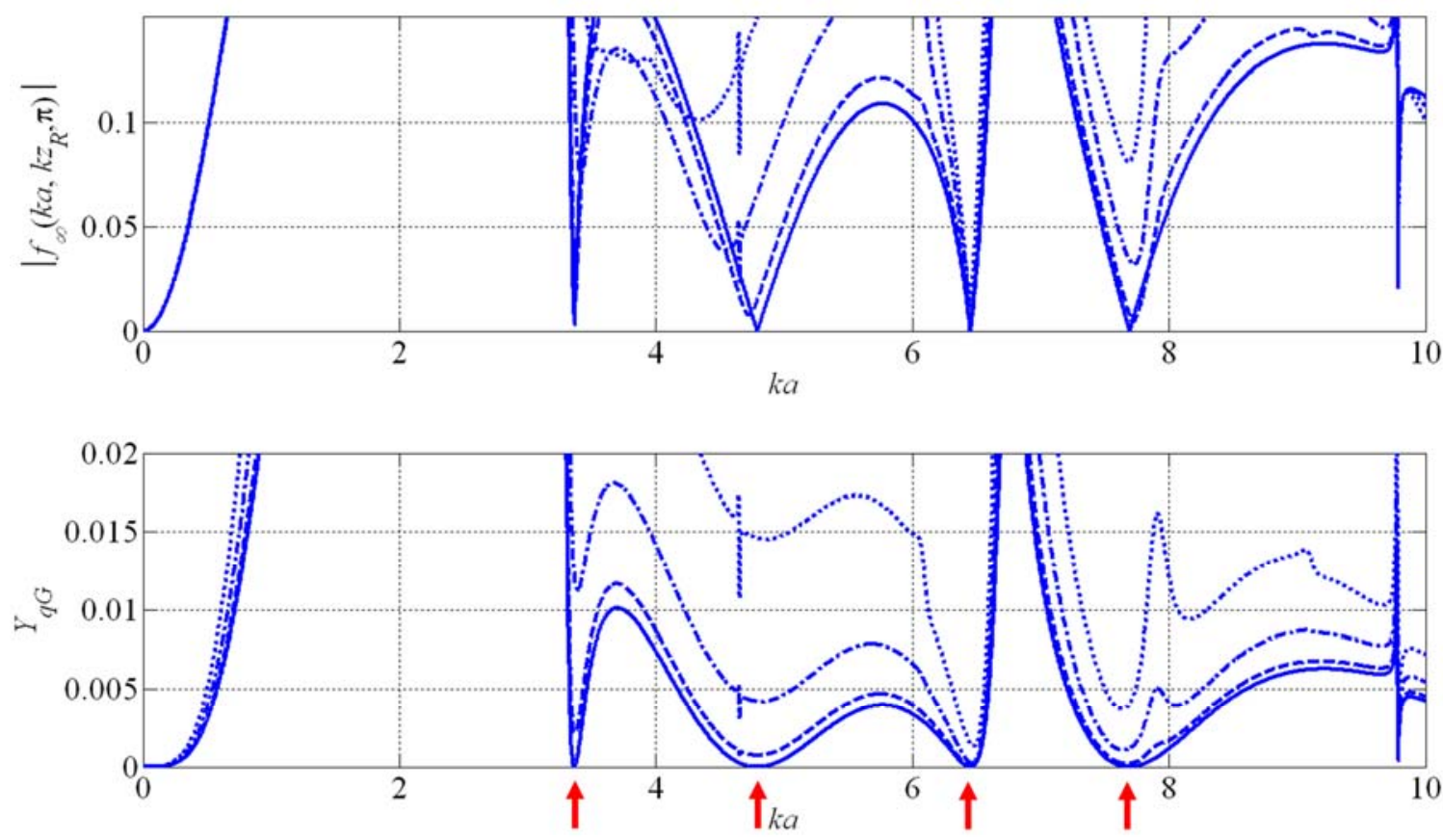

1

2 Fig. 2. (Color online) The plots for the backscattering form-function (top panel) and

3 radiation force function (bottom panel), for a polymethylmetacrylate (PMMA) elastic

4 sphere for $k w_{0}=0.1$ (solid line), $k w_{0}=1$ (long-dashed line), $k w_{0}=1.5$ (dashed-dotted

5 line), and $k w_{0}=2$ (dotted line). The arrows along the $k a$ axis in the bottom panel point to

6 the zeros of $Y_{q G}$ that occur at the minima-resonances of the elastic sphere. 


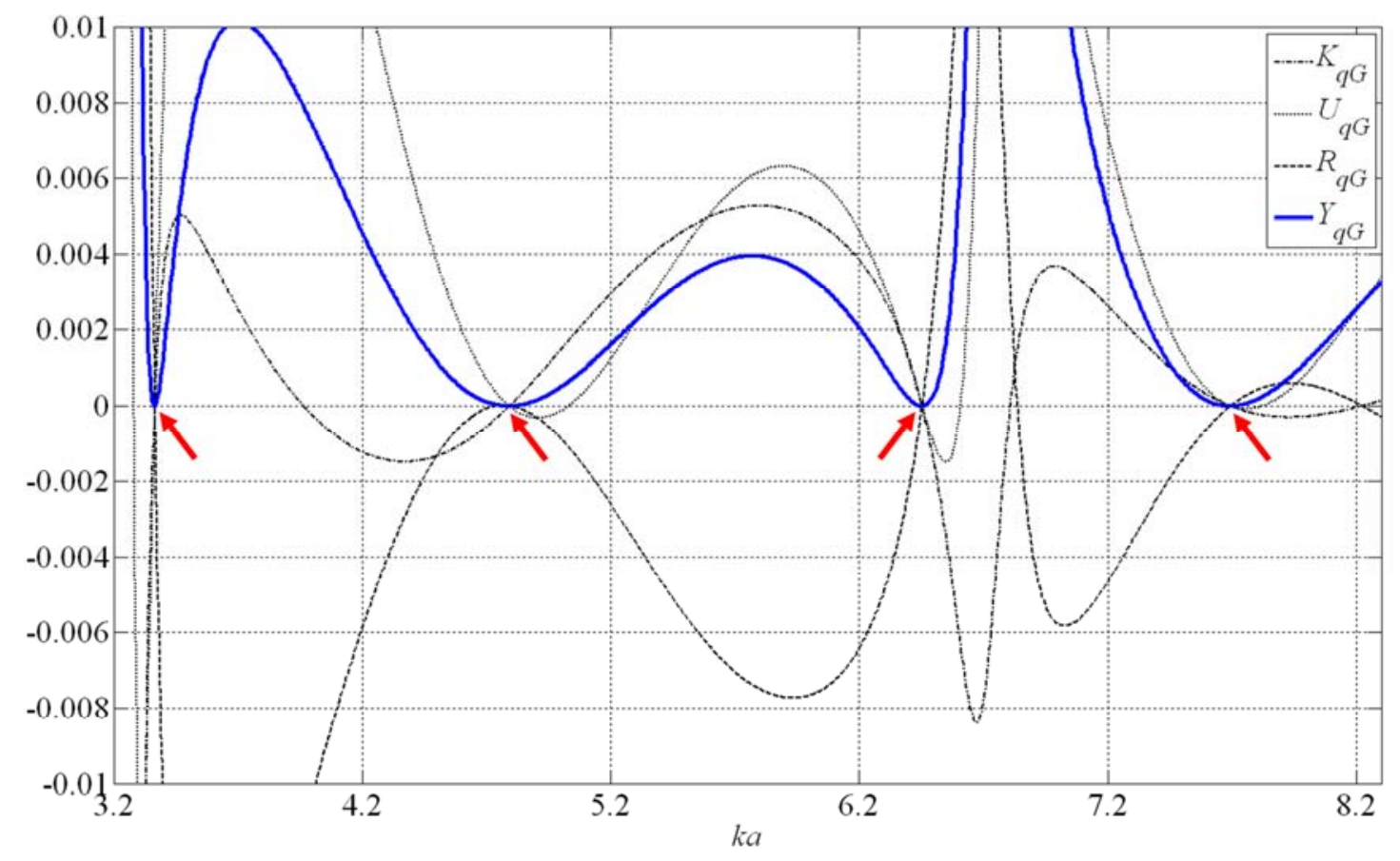

2 Fig. 3. (Color online) The plots for the the components $K_{q G}, U_{q G}, R_{q G}$ as well as $Y_{q G}$ for

3 an elastic PMMA sphere for $k w_{0}=0.1$. It is noticed that all the three components, namely

4 the kinetic energy density, the potential energy density as well as the momentum flux

5 density vanish simultaneously at the selected $k a$ values for the nulls.

6

7

8 
(a) $k w_{0}-0.1: k a-3.366$

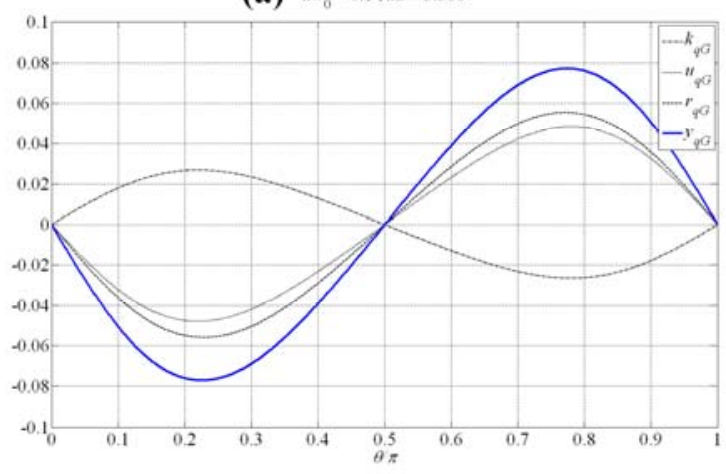

(c) $k w_{0}-0.1: k a=6.456$

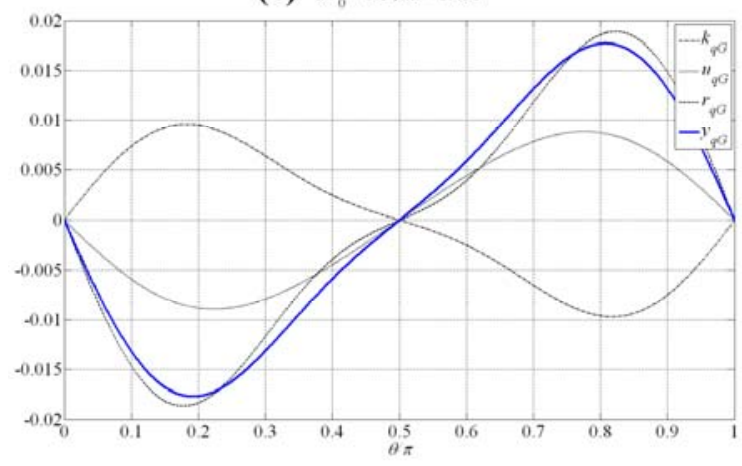

(b)

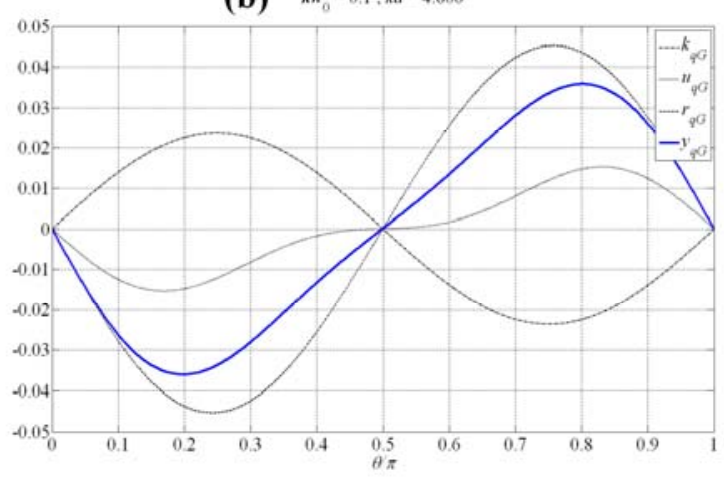

(d) $k w_{0}-0.1 ; k a-7.691$

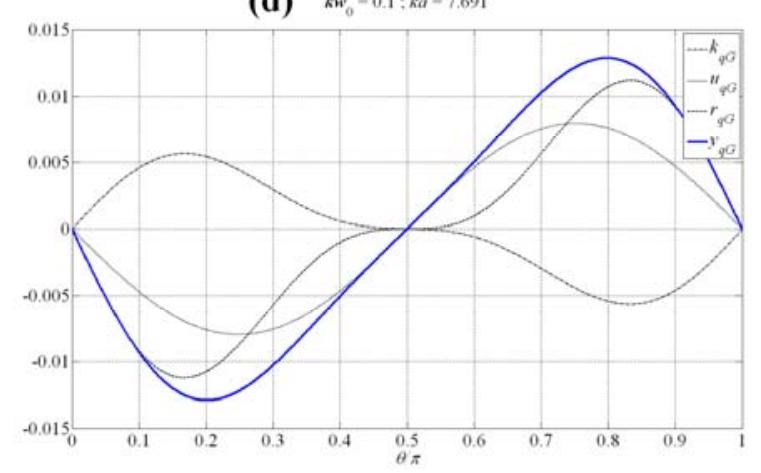

2 Fig. 4. (Color online) The plots for the density functions for an elastic PMMA sphere for

$3 k w_{0}=0.1$ at the zeros of $Y_{q G}$ (pointed to by arrows in Fig. 3). In all cases, all the density

4 functions including $y_{q G}(\theta)$ exhibit an anti-symmetric behavior with respect to the

5 direction $\theta / \pi=0.5$.

6 


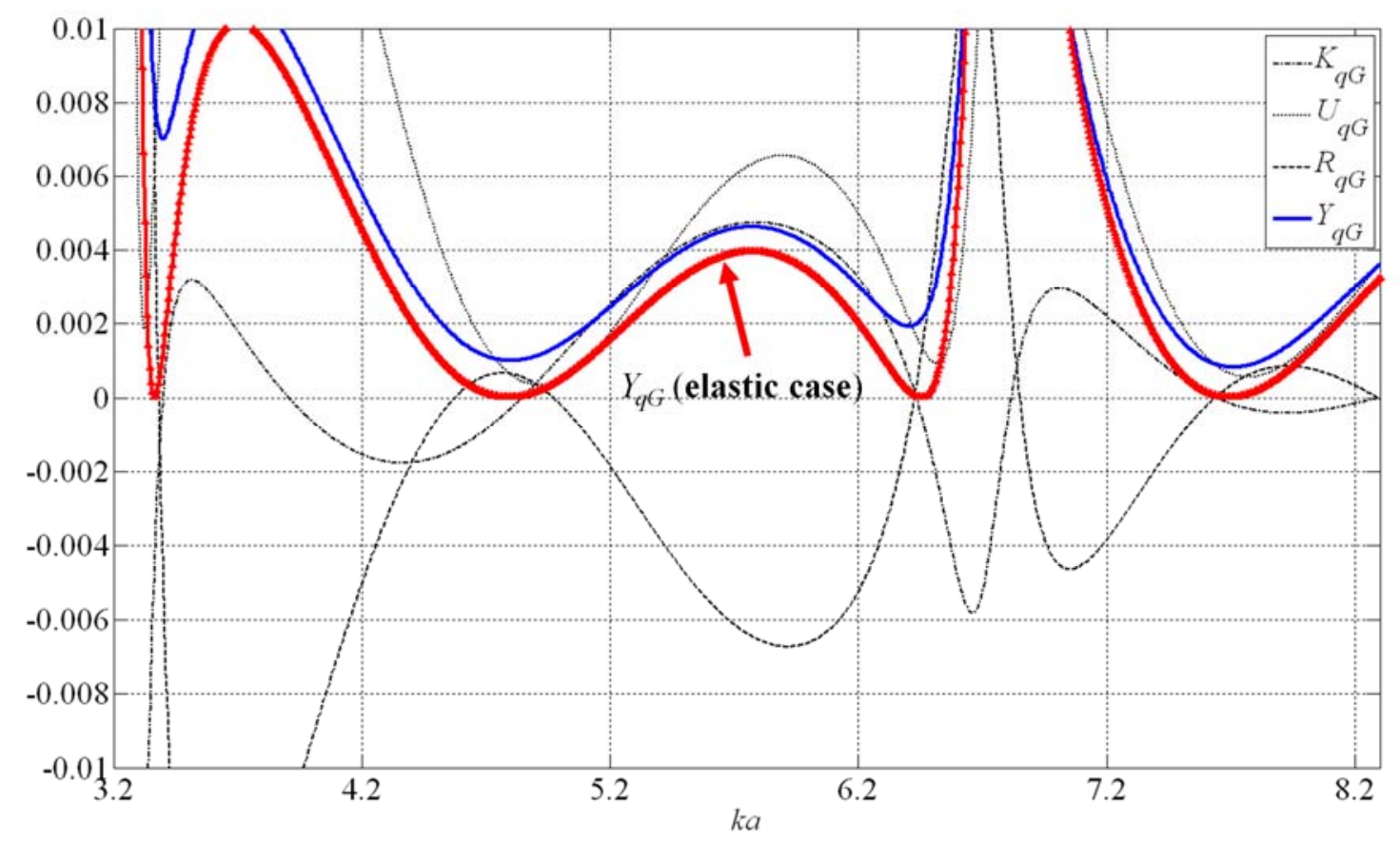

2 Fig. 5. (Color online) The same as in Fig. 3, however the PMMA sphere is viscoelastic

3 (sound absorptive). The (red) curve with triangles $(\Delta)$ corresponds to the case of no-

4 absorption and is added for convenience.

5

6

7

8 


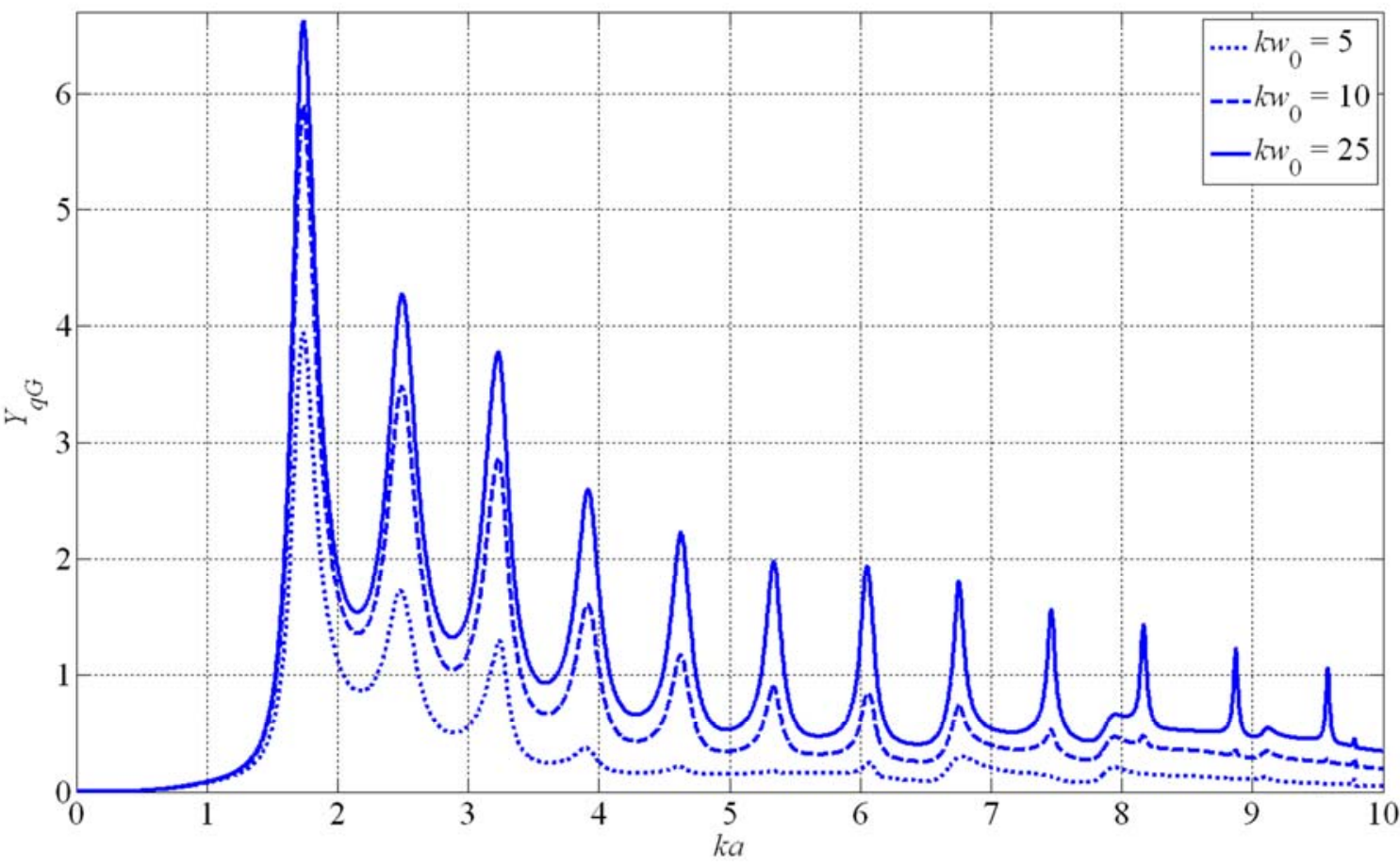

1

2 Fig. 6. (Color online) The $Y_{q G}$ plots for a PMMA elastic sphere in water for $k w_{0}=5,10$

3 and 25, respectively. The (quasi)plane wave limit is reached for $\geq 25$.

4 


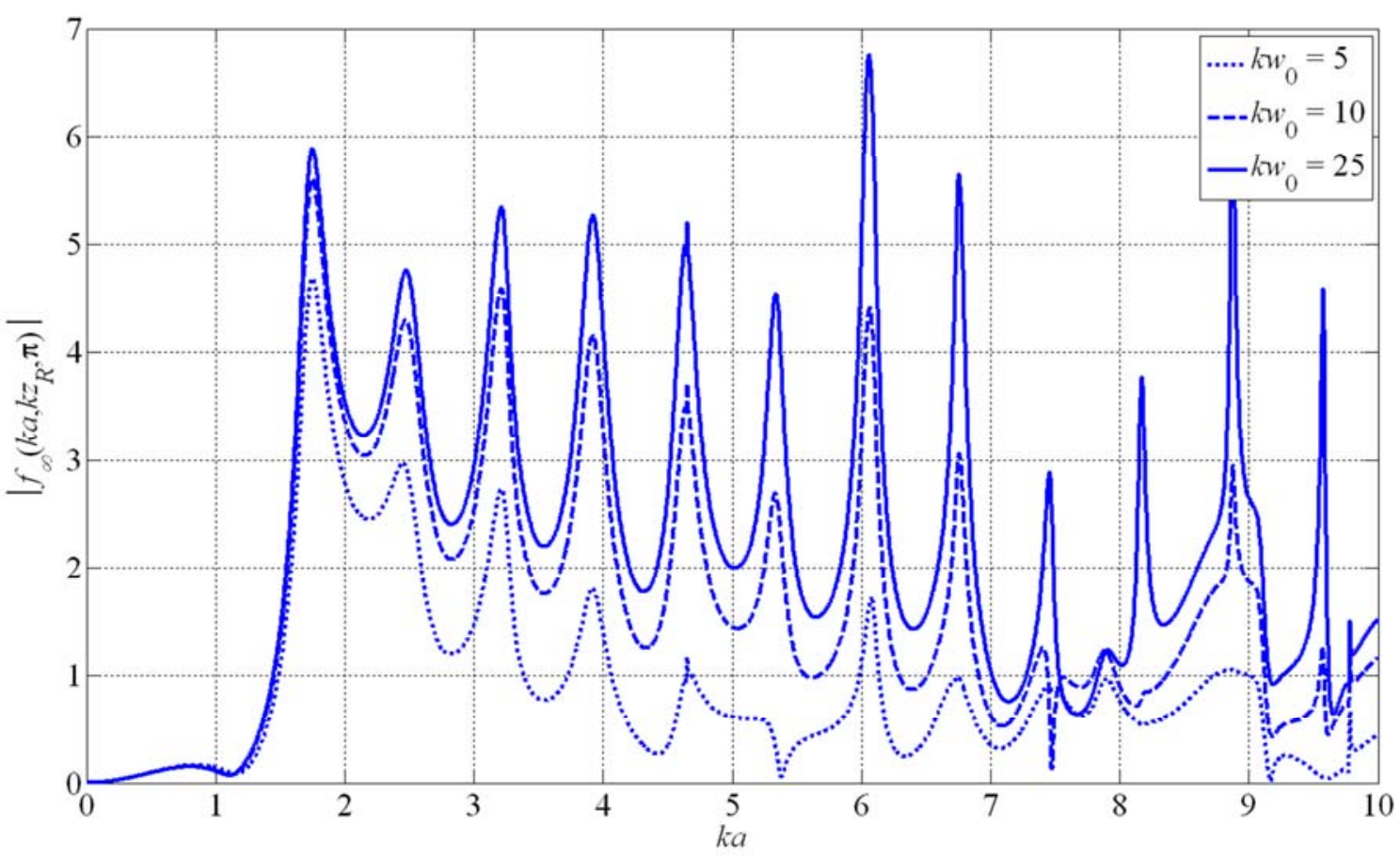

2 Fig. 7. (Color online) The magnitude of the backscattering form function $3\left|f_{\infty}\left(k a, k z_{R}, \pi\right)\right|$ plots for $k w_{0}=5,10$ and 25 , respectively.

4

5

6

7

8

9

10

11

12

13

14 


\section{References}

[1] J.B. Keller, J.F.C. Karal, Surface Wave Excitation and Propagation, J Appl Phys, 31 (1960) 1039-1046.

[2] G.A. Deschamps, Gaussian beam as a bundle of complex rays, Electron. Lett., 7 (1971) 684-685.

[3] J.B. Keller, W. Streifer, Complex Rays with an Application to Gaussian Beams, J. Opt. Soc. Am., 61 (1971) 40-43.

[4] L.B. Felsen, Evanescent waves, J. Opt. Soc. Am., 66 (1976) 751-760.

[5] M. Couture, P.A. Belanger, From Gaussian beam to complex-source-point spherical wave, Physical Review A, 24 (1981) 355-359.

[6] A.N. Norris, Complex point-source representation of real point sources and the Gaussian beam summation method, J. Opt. Soc. Am. A, 3 (1986) 2005-2010.

[7] A.N. Norris, T.B. Hansen, Exact complex source representations of time-harmonic radiation, Wave Motion, 25 (1997) 127-141.

[8] F.G. Mitri, Quasi-Gaussian electromagnetic beams, Physical Review A, 87 (2013) 035804.

[9] O.A. Sapozhnikov, An exact solution to the Helmholtz equation for a quasi-Gaussian beam in the form of a superposition of two sources and sinks with complex coordinates, Acoust Phys, 58 (2012) 41-47.

[10] M. Azarpeyvand, M. Azarpeyvand, Acoustic radiation force on a rigid cylinder in a focused Gaussian beam, J Sound Vib, 332 (2013) 2338-2349.

[11] F.G. Mitri, Interaction of an acoustical Quasi-Gaussian beam with a rigid sphere: linear axial scattering, instantaneous force, and time-averaged radiation force, IEEE Transactions on Ultrasonics, Ferroelectrics and Frequency Control, 59 (2012) 23472351.

[12] D.E. Chimenti, J.G. Zhang, S. Zeroug, L.B. Felsen, Interaction of Acoustic Beams with Fluid-Loaded Elastic Structures, J Acoust Soc Am, 95 (1994) 45-59.

[13] L. Schmerr, J.-S. Song, Ultrasonic Nondestructive Evaluation Systems: Models and Measurements, Springer, 2007.

[14] F.G. Mitri, Acoustic radiation force due to incident plane-progressive waves on coated spheres immersed in ideal fluids, Eur Phys J B, 43 (2005) 379-386.

[15] F.G. Mitri, Erratum to: Acoustic radiation force due to incident plane-progressive waves on coated spheres immersed in ideal fluids, The European Physical Journal B - Condensed Matter and Complex Systems, 76 (2010) 185-185.

[16] F.G. Mitri, Z.E.A. Fellah, The mechanism of the attracting acoustic radiation force on a polymer-coated gold sphere in plane progressive waves, European Physical Journal E, 26 (2008) 337-343.

[17] F.G. Mitri, Calculation of the acoustic radiation force on coated spherical shells in progressive and standing plane waves, Ultrasonics, 44 (2006) 244 -258.

[18] K. Yosioka, Y. Kawasima, Acoustic radiation pressure on a compressible sphere, Acustica, 5 (1955) 167-173.

[19] T. Hasegawa, K. Yosioka, Acoustic radiation force on a solid elastic sphere, J Acoust Soc Am, 46 (1969) 1139-1143.

[20] T. Hasegawa, T. Kido, C.W. Min, T. Iizuka, C. Matsuoka, Frequency dependence of the acoustic radiation pressure on a solid sphere in water, Acoustical Science and Technology, 22 (2001) 273-282. 
[21] V.M. Ayres, G.C. Gaunaurd, Acoustic resonance scattering by viscoelastic objects, J Acoust Soc Am, 81 (1987) 301-311.

[22] A. Pierce, Acoustics: An Introduction to Its Physical Principles and Applications, Acoustical Society of America, 1994.

[23] T. Kido, T. Hasegawa, N. Okamura, Mechanisms for the attracting acoustic radiation force on a rigid sphere placed freely in a spherical sound field, Acoustical Science and Technology, 25 (2004) 439-445.

[24] T. Hasegawa, M. Ochi, K. Matsuzawa, Acoustic radiation pressure on a rigid sphere in a spherical wave field, J Acoust Soc Am, 67 (1980) 770-773.

[25] T. Hasegawa, M. Ochi, K. Matsuzawa, Acoustic radiation force on a solid elastic sphere in a spherical wave field, J Acoust Soc Am, 69 (1981) 937-942.

[26] X. Chen, R.E. Apfel, Radiation force on a spherical object in an axisymmetric wave field and its application to the calibration of high-frequency transducers, J Acoust Soc Am, 99 (1996) 713-724.

[27] J. Lee, K.K. Shung, Radiation forces exerted on arbitrarily located sphere by acoustic tweezer, J Acoust Soc Am, 120 (2006) 1084-1094.

[28] J. Lee, S.-Y. Teh, A. Lee, H.H. Kim, C. Lee, K.K. Shung, Single beam acoustic trapping, Applied Physics Letters, 95 (2009) 073701-073703.

[29] P.L. Marston, Axial radiation force of a Bessel beam on a sphere and direction reversal of the force, J Acoust Soc Am, 120 (2006) 3518-3524.

[30] F.G. Mitri, Langevin acoustic radiation force of a high-order Bessel beam on a rigid sphere, Ieee T Ultrason Ferr, 56 (2009) 1059-1064.

[31] F.G. Mitri, Negative Axial Radiation Force on a Fluid and Elastic Spheres Illuminated by a High-Order Bessel Beam of Progressive Waves, Journal of Physics A - Mathematical and Theoretical, 42 (2009) 245202.

[32] P.L. Marston, Radiation force of a helicoidal Bessel beam on a sphere, The Journal of the Acoustical Society of America, 125 (2009) 3539.

[33] A.K. Miri, F.G. Mitri, Acoustic Radiation Force on a Spherical Contrast Agent Shell Near a Vessel Porous Wall - Theory, Ultrasound in Medicine \& Biology, 37 (2011) 301-311.

[34] L.S. Schuetz, W.G. Neubauer, Acoustic reflection from cylinders---nonabsorbing and absorbing, The Journal of the Acoustical Society of America, 62 (1977) 513517.

[35] F.G. Mitri, Z.E.A. Fellah, J.Y. Chapelon, Acoustic backscattering form function of absorbing cylinder targets (L), J Acoust Soc Am, 115 (2004) 1411-1413.

[36] F.G. Mitri, Acoustic radiation force acting on absorbing spherical shells, Wave Motion, 43 (2005) 12-19.

[37] F.G. Mitri, Acoustic radiation force on a sphere in standing and quasi-standing zeroorder Bessel beam tweezers, Annals of Physics, 323 (2008) 1604-1620.

[38] L.K. Zhang, P.L. Marston, Geometrical interpretation of negative radiation forces of acoustical Bessel beams on spheres, Phys Rev E, 84 (2011) 035601.

[39] G.T. Silva, An expression for the radiation force exerted by an acoustic beam with arbitrary wavefront (L), The Journal of the Acoustical Society of America, 130 (2011) 3541-3544.

[40] F.G. Mitri, G.T. Silva, Off-axial acoustic scattering of a high-order Bessel vortex beam by a rigid sphere, Wave Motion, 48 (2011) 392-400. 
1 [41] G.T. Silva, Off-axis scattering of an ultrasound Bessel beam by a sphere, Ieee T 2 Ultrason Ferr, 58 (2011) 298-304.

3 [42] F.G. Mitri, Generalized theory of resonance excitation by sound scattering from an 4 elastic spherical shell in a nonviscous fluid, IEEE Transactions on Ultrasonics, $5 \quad$ Ferroelectrics and Frequency Control, 59 (2012) 1781-1790.

6 [43] F.G. Mitri, Arbitrary scattering of an acoustical high-order Bessel trigonometric 7 (non-vortex) beam by a compressible soft fluid sphere, Ultrasonics, $8 \quad$ http://dx.doi.org/10.1016/j.ultras.2012.12.008 (2013).

9 [44] D. Baresch, J.-L. Thomas, R. Marchiano, Three-dimensional acoustic radiation force 10 on an arbitrarily located elastic sphere, The Journal of the Acoustical Society of 11 America, 133 (2013) 25-36.

12 [45] G.T. Silva, J.H. Lopes, F.G. Mitri, Off-axial acoustic radiation force of repulsor and 13

14 tractor Bessel beams on a sphere Ieee T Ultrason Ferr, accepted - in press (2013). 